\title{
Catalytic Locomotion of Core-Shell Nanowire Motors
}

\section{Journal Article}

\section{Author(s):}

Jang, Bumjin; Wang, Wei; Wiget, Samuel; Petruska, Andrew J.; Chen, Xiangzhong; Hu, Chengzhi; Hong, Ayoung (D); Folio, David; Ferreira, Antoine; Pané, Salvador; Nelson, Bradley J.

Publication date:

2016-11

\section{Permanent link:}

https://doi.org/10.3929/ethz-b-000123251

\section{Rights / license:}

In Copyright - Non-Commercial Use Permitted

\section{Originally published in:}

ACS Nano 10(11), https://doi.org/10.1021/acsnano.6b04224 


\title{
Catalytic Locomotion of Core-Shell Nanowire Motors
}

Bumjin Jang, ${ }^{\dagger}$ Wei Wang, ${ }^{\ddagger}{ }^{\circledR}$ Samuel Wiget,$^{\dagger}$ Andrew J. Petruska,$^{\dagger}$ Xiangzhong Chen,$^{\dagger}$ Chengzhi Hu, ${ }^{\dagger}$ Ayoung Hong, ${ }^{\dagger}$ David Folio,${ }^{\S}$ Antoine Ferreira, ${ }^{\S}$ Salvador Pané, ${ }^{* \dagger}$ and Bradley J. Nelson ${ }^{*}{ }^{\dagger}$

\author{
${ }^{\dagger}$ Institute of Robotics and Intelligent Systems, ETH Zurich, Zurich, CH-8092, Switzerland \\ IShenzhen Key Laboratory for Advanced Materials, School of Material Sciences and \\ Engineering, Shenzhen Graduate School, Harbin Institute of Technology, University Town, \\ Shenzhen 518055, China
}

ITCenter for Soft and Living Matters, Institute of Basic Sciences, Ulsan, Korea

${ }^{\S}$ INSA Centre Val de Loire, Universite d'Orléans, PRISME EA 4229, Bourges, France.

\begin{abstract}
We report the partial core-shell nanowire motors. These nanowires are fabricated using our previously developed electrodeposition-based technique, and their catalytic locomotion in the presence of $\mathrm{H}_{2} \mathrm{O}_{2}$ is investigated. Unlike conventional bimetallic nanowires that are selfelectroosmotically propelled, our $\mathrm{Au} / \mathrm{Ru}$ core-shell nanowires show both a noticeable decrease in rotational diffusivity and increase in motor speed with nanowire length. Numerical modelling based on self-electroosmosis attributes the decreases in rotational diffusivity to the formation of toroidal vortices at the nanowire tail, but fails to explain the speed increase with length. To reconcile this inconsistency, we propose a combined mechanism of self-diffusiophoresis and
\end{abstract}


electroosmosis based on the oxygen gradient produced by catalytic shells. This mechanism successfully explains not only the peculiar speed increase of $\mathrm{Au} / \mathrm{Ru}$ core-shell nanomotors with length, but also the large variation in speeds among $\mathrm{Au} / \mathrm{Ru}, \mathrm{Au} / \mathrm{Rh}$ and $\mathrm{Rh} / \mathrm{Au}$ core-shell nanomotors. The possible contribution of diffusiophoresis to an otherwise well-established electroosmotic mechanism sheds light on future designs of nanomotors, at the same time highlighting the complex nature of nanoscale propulsion.

KEYWORDS: Electrodeposition, core-shell nanowires, catalytic nanomotors, selfelectroosmosis, self-diffusiophoresis, net propulsive force

Fifteen years ago, Whitesides et al. pioneered self-propelled catalytic millimeter-scale ships ${ }^{1}$ triggering the development of synthetic micro- and nanomotors. These devices move autonomously in the presence of a fuel solution by converting chemical to mechanical energy. ${ }^{2}$ Most reported catalytic micro- and nanomotors are driven by the decomposition of hydrogen peroxide, although examples using glucose and urea as fuel have been reported recently. ${ }^{3-6}$ Several applications for these miniaturized motors have been demonstrated such as electronic circuit healing, ${ }^{7}$ biosensing,,${ }^{4} 8$ nanolithography, ${ }^{9}$ microfluidic pumping,${ }^{10}$ nanocargo transport, ${ }^{11}$ and environmental remediation (for details, see recent review articles ${ }^{12-17}$ ). Most of these applications require precise control over the speed and directionality of the motors, ${ }^{18}$ which are highly dependent on motor design parameters, such as geometry, size and composition. Understanding the mechanisms involved in the propulsion of catalytic micro- and nanomotors as a function of their design is crucial for controlling their motion. Different propulsion mechanisms have been identified, such as bubble propulsion, self-electroosmosis, selfdiffusiophoresis, and interfacial tension. ${ }^{17}$ In the first mechanism, bubbles are formed on the catalytic surface and impart momentum to the motor. For example, tubular microjets are driven 
by oxygen bubble propulsion, caused by the decomposition of $\mathrm{H}_{2} \mathrm{O}_{2}$ at the inner catalytic surface. ${ }^{19,20}$ While bubble propulsion seems the most intuitive way to explain the motion of these motors, this mechanism cannot be used to rationalize, for instance, the motion of bimetallic structures such as $\mathrm{Pt} / \mathrm{Au}$ or $\mathrm{Cu} / \mathrm{Pt}$ nanowires (NWs) in $\mathrm{H}_{2} \mathrm{O}_{2}$ and $\mathrm{I}_{2}$ solutions, respectively. ${ }^{21,}{ }^{22}$ For bimetallic NWs, self-electroosmosis is generally the most accepted mechanism. ${ }^{22,}{ }^{23}$ In this case, a self-induced electric field is generated by redox reactions occurring on the two different metallic segments. The motion of other micro- and nanomotors has been explained in terms of self-diffusiophoresis. Several efforts suggest that micro- and nanoparticles asymmetrically coated with $\mathrm{Pt}$ move due to an $\mathrm{O}_{2}$ concentration gradient caused by the asymmetric decomposition of $\mathrm{H}_{2} \mathrm{O}_{2}$ along the body of the motor. ${ }^{24-28}$ Recent investigations evaluating more complex motor architectures indicate a concomitant occurrence of mechanisms. ${ }^{29-31}$

Among all micro- and nanomotor structures, NWs are particularly appealing due to their unique physical properties. ${ }^{32}$ Additionally, NWs can be compartmentalized in several functional modules by means of segmentation ${ }^{33,34}$ or coaxial lithography $y^{35}$ to increase their capabilities. For instance, the trajectory of a catalytic NW motor consisting of $\mathrm{Pt} / \mathrm{Ni} / \mathrm{Au} / \mathrm{Ni} / \mathrm{Au}$ segments can be controlled by means of magnetic fields. ${ }^{36}$ However, interfacing certain building blocks can be detrimental for several applications. For example, the combination of two dissimilar metallic segments such as Au and Ni can lead to an increase of the corrosion rate of the magnetic part due to galvanic coupling. ${ }^{37}$ Also, the interaction of certain materials (e.g. Ni) with the surrounding environment (e.g., biological or aquatic media) must be avoided to prevent undesired leakage of toxic ions. Alternatively, a core-shell arrangement can provide an inert housing to shield certain components from the surrounding environment. Core-shell NWs have been manufactured using a wide variety of methods, which usually require two or more steps ${ }^{38-43}$ However, a purely core- 
shell structure, by definition, cannot become a motor due to the inherent absence of broken symmetry in composition. By comparison, a conventional NW motor (e.g. Au/Pt bimetallic rods in $\mathrm{H}_{2} \mathrm{O}_{2}$ ) has both of their surfaces exposed to the solution and thus propels. A partial core-shell nanomotor that can shelter the active components while generating asymmetric propulsion is, therefore, desired but challenging.

In this work, we synthesized partial core-shell nanomotors with one side of the core exposed to solutions, by capitalizing on a recently developed method that allows for the batch fabrication of freestanding NWs based on template-assisted electrodeposition and wafer bonding. As the NWs were self-supported on the wafer after template removal, a second electrodeposition step produced a core-shell architecture. These core-shell nanowires were, then, sonicated off from the substrate, exposing the first (therefore, core) material to the solution at the breaking point. This developed method can be adopted to fabricate core-shell NWs with various material combinations. Here, we synthesized different types of bimetallic core-shell NWs, such as $\mathrm{Au} / \mathrm{Ru}$, $\mathrm{Rh} / \mathrm{Au}$ and $\mathrm{Au} / \mathrm{Rh}$ (core/shell), and investigated their motion in $\mathrm{H}_{2} \mathrm{O}_{2}$ solutions. Importantly, abnormal behaviors were found, including a speed increase with the length of $\mathrm{Au} / \mathrm{Ru}$ core-shell NWs and a large speed difference among $\mathrm{Au} / \mathrm{Ru}, \mathrm{Rh} / \mathrm{Au}$ and $\mathrm{Au} / \mathrm{Rh}$. These behaviors are not observed for conventional bimetallic NWs, and, therefore, cannot be accounted for solely by self-electroosmosis. To understand these unusual trends, we propose a combined mechanism of self-diffusiophoresis and electroosmosis based on the oxygen gradient produced by catalytic shells, which successfully explain all experimental observations.

\section{Results/Discussion}

Design and fabrication of the Au/Ru core-shell NWs. The fabrication process is illustrated in Figure 1a. First, an anodic aluminum oxide (AAO) membrane was transferred onto a Si substrate 
via the $\mathrm{Au} / \mathrm{Au}$ compressive bonding technique previously developed in our group. ${ }^{44} \mathrm{Next}, \mathrm{Au}$ was electrodeposited into the pores of the AAO. The AAO was then chemically etched to obtain a freestanding Au NW array on the Si substrate. Ru was then electrochemically deposited on the freestanding Au NW array, resulting in the formation of a core-shell structure. The strong adhesion between the freestanding array and the $\mathrm{Si}$ substrate is able to withstand the electroosmotic and fluidic forces exerted on the NWs during Ru electrodeposition. The freestanding NWs must be kept in solution to prevent drying, because the surface tension forces during drying will cause severe NW aggregation ${ }^{45}$ and result in a non-uniform deposition of the Ru shell. After the Ru deposition, the NWs were released by sonication.

The scanning electron microscope (SEM) image in Figure 1b, obtained using a backscattered electron (BSE) detector, shows a typical Au/Ru core-shell NW with an approximate diameter and length of $350 \mathrm{~nm}$ and $2.5 \mu \mathrm{m}$, respectively, where the length varies with the deposition time of the Au core. Since the intensity of the BSE signal is proportional to the atomic number, the bright area and the dark area can be assigned to $\mathrm{Au}$ and $\mathrm{Ru}$, respectively. The $\mathrm{Au}$ core appears only at the base (left end) as a consequence of being released from the Si substrate. No obvious defects are observed in the Au/Ru core-shell NW structure as is seen in the highangle annular dark field (HAADF) scanning transmission electron microscopy (STEM) image (Figure 1c). The corresponding energy dispersive X-ray (EDX) mapping verifies that the coreshell NW is composed of $\mathrm{Au}$ and Ru (Figure 1d). For a better understanding of the Ru distribution, we then selectively etched the Au core in Au etchant. Representative SEM and STEM images (Figure 1e,f) show that the remaining structure after etching is a Ru shell. The Ru shell has an approximate diameter of $350 \mathrm{~nm}$, which is similar to that of a Au/Ru core-shell NW. A smooth surface of the nanoshell without holes or cracks on the rod is observed and confirms 
the uniform distribution and the conformal growth of the Ru shell. Subsequent EDX mapping verifies that the nanoshell solely consists of Ru (Figure 1g). The structures of a conventional bimetallic nanorod, a purely core-shell nanowire, and our partial core-shell nanowire are given in Fig S1 to highlight the difference in their structures. We note that "core-shell NW" is used to refer to our partially core-shell structure from here on for the sake of simplicity.

Numerical modeling of electroosmotic mechanism of core-shell NWs. Electroosmosis is the main driving force for locomotion based on surface redox reactions. ${ }^{46,47}$ The redox reaction consists of an oxidation reaction and reduction reaction as illustrated in Figure 2a. Protons, oxygen molecules, and electrons are generated in the oxidation process at the anode (Au) by decomposing $\mathrm{H}_{2} \mathrm{O}_{2}$. The generated protons travel by convection, diffusion, and migration to the cathode $(\mathrm{Ru})$, where they react with electrons, oxygen, and $\mathrm{H}_{2} \mathrm{O}_{2}$ molecules to produce $\mathrm{H}_{2} \mathrm{O}$ in the reduction process. As a consequence of this redox reaction, protons are asymmetrically distributed around the nanomotor, and an asymmetric electric field is developed. This selfgenerated electric field causes protons to migrate inside the electrical double layer that naturally forms when the nanomotor is immerged in a water solution. Proton migration leads to an electroosmotic flow, which induces the motion of the nanomotor.

The influence of the electroosmotic mechanism on the locomotion of our core-shell nanomotor was simulated using the commercial multiphysics simulation package COMSOL (5.1). ${ }^{48}$ A number of important results were obtained from the simulation, and served to guide our experiments. First, Figure S2a shows that the proton concentration around the Au/Ru coreshell NW is highly asymmetric, higher near $\mathrm{Au}$ end and lower near $\mathrm{Ru}$ end, resulting in an asymmetric electric potential distribution as seen in Figure S2b. Consequently, the fluid flows 
from the anode to the cathode, hence propulsion of the $\mathrm{Au} / \mathrm{Ru}$ core-shell $\mathrm{NW}$ with its anode end $\mathrm{Au}$ ) first. The simulation also reveals that the fluid speed is highest at the interface between the cathode and anode of the core-shell NW, where the majority of the electroosmotic flow occurs.

One important feature we noticed in our simulation (Figure 2b) was that at the end opposite the anode, the electroosmotic flow can be viewed as a combination of two components: the flow from the anode to cathode and the flow from the surrounding solution to the anode. We further investigated how the length of $\mathrm{Au} / \mathrm{Ru}$ core-shell $\mathrm{NW}$ affects the convergent flow. When the length is sufficiently small ( $500 \mathrm{~nm}$, Figure $2 \mathrm{~b}(\mathrm{i}))$, the electric field generated at the anode dominates the overall electroosmotic flow (Figure S2c), and the disturbance to the fluid flow at the tip of the cathode is negligible. At approximately $1.5 \mu \mathrm{m}$, a flow reversal at the tip occurs, which is caused by the migration of protons from the surrounding solution to the cathode (Figure $2 b$ (ii)). The reverse flow creates a toroidal vortex near the surface of the NW tip. Further increases in length increase the size of the vortex (Figure $2 b$ (iii), (iv)). The vortex causes an increase in fluidic drag at the tail of the NW, as shown by the negative fluidic speed at the cathode end of the NW (Figure S2d).

Lastly, the nanomotor speeds were calculated from the fluid velocities around the coreshell NWs using the Solomentsev and Anderson model, and their speeds as a function of length of the core-shell NW is plotted in Figure $2 c .{ }^{48}$ Our simulation result shows a clear decrease in motor speed as the core-shell NW length increases, which can be attributed to an increase in drag force with increasing NW length, since a no-slip boundary condition was chosen for the nanomotor surface in the simulation. In the following section we will describe how our experiments confirm and violate these seemingly straightforward simulation results, and how such conflict leads to a better understanding of the propulsion mechanism of core-shell NWs. 
Catalytic propulsion of the $\mathrm{Au} / \mathrm{Ru}$ core-shell NWs. The catalytic locomotion of the $\mathrm{Au} / \mathrm{Ru}$ core-shell NWs (approximate length of $2.5 \mu \mathrm{m}$ ) in $\mathrm{H}_{2} \mathrm{O}_{2}$ solution was investigated at various concentrations $(0,0.1,0.3,2.5,5,10$ and $20 \mathrm{wt} \%)$. The speeds of the core-shell NWs were found to increase with the concentration of $\mathrm{H}_{2} \mathrm{O}_{2}$ (Figure 3a), showing the maximum speed of 41 $\pm 7.6 \mu \mathrm{m} / \mathrm{s}$ at $20 \mathrm{wt} \% \mathrm{H}_{2} \mathrm{O}_{2}$. This trend is consistent with both our simulation results and previous findings. ${ }^{49-52}$ A representative core-shell NW trajectory is shown in Figure 3a (i). A large displacement is observed over $4.55 \mathrm{sec}$ in the presence of $\mathrm{H}_{2} \mathrm{O}_{2}(5 \mathrm{wt} \%)$ in contrast to the control experiment, which was conducted in the absence of $\mathrm{H}_{2} \mathrm{O}_{2}$ and shows Brownian motion with a very small displacement (Figure 3a (ii)).

To systematically understand the locomotion, we studied the dynamic behavior of the core-shell NW with different lengths $(\approx 1.5,2.5$ and $3.5 \mu \mathrm{m})$. For this, at least 10 different motion trajectories with the total duration of about $6 \mathrm{sec}$ were recorded for each length. From the trajectories, we calculated mean-square displacement (MSD) up to the time interval of $0.23 \mathrm{sec}$, using:

$\Delta L^{2}(t) \equiv<[\mathbf{r}(t)-\mathbf{r}(0)]^{2}>$

Also, mean-square angular displacement (MSAD) was calculated up to the time interval of $1 \mathrm{sec}$, using:

$\Delta \theta^{2}(t) \equiv<[\boldsymbol{\theta}(t)-\boldsymbol{\theta}(0)]^{2}>$

where $\mathbf{r}(t)=(x(t), y(t))$

Then, the MSD and MSAD values were averaged. Translational diffusivity $\left(D_{t}\right)$, translational velocity $(v)$, rotational diffusivity $\left(D_{r}\right)$ and rotational velocity $(\omega)$ can be extracted by fitting the 
MSD and MSAD values in these very short time intervals with quadratic functions as follows. First, $D_{t}$, and $v$ were extracted from the averaged MSD values with ${ }^{29,53}$ :

$\Delta L^{2}(t)=4 D_{t} \Delta t+v^{2} \Delta t^{2}$

Also, $D_{r}$ and $\omega$ were extracted from the averaged MSAD values with ${ }^{53}$ :

$\Delta \theta^{2}(\mathrm{t})=2 D_{r} \Delta t+\omega^{2} \Delta t^{2}$

The averaged MSD (or MSAD) data, and the fitted lines are plotted as scatter and line type, respectively; MSD in Figure $3 \mathrm{~b}$ and MSAD in Figure 3c.

For comparison, the translational diffusivity $D_{t 0}$ and the rotational diffusivity $D_{r 0}$ of passive Brownian motion (i.e. in the absence of $\mathrm{H}_{2} \mathrm{O}_{2}$ ) of our core-shell NW are calculated by the Einstein relation:

$D_{t 0}=\frac{k_{B} T}{f_{t}^{\|}}$

$D_{r 0}=\frac{k_{B} T}{f_{r}^{\perp}}$

where $k_{B}$ is the Boltzmann constant, $T$ is the absolute temperature, $f_{t}^{\|}$is the translational frictional drag force parallel to the long axis, and $f_{r}^{\perp}$ is the rotational frictional drag force about the short axis of NW with a length $(L)$ and a diameter $(d)$ in a viscous media $\eta$. The drag terms $f_{t}^{\| l}$ and $f_{r}^{\perp}$ are defined as: $:^{54}$

$f_{t}^{\|}=\frac{2 \pi \eta L}{\ln (L / d)-0.207+\frac{0.980}{(L / d)}-\frac{0.133}{(L / d)^{2}}}$

$f_{r}^{\perp}=\frac{\pi \eta L^{3}}{3\left(\ln (L / d)-0.662+\frac{0.917}{(L / d)}-\frac{0.05}{(L / d)^{2}}\right)}$

Table 1 shows that the experimental values of $D_{t}$ and $D_{r}$ are higher than the theorical values of $D_{t 0}$ and $D_{r}$ probably due to slight variations in diameters and lengths from sample by 
sample. Nevertheless, the translational diffusivity $D_{t}$, rotational diffusivity $D_{r}$, and rotational velocity $\omega$ decrease with increasing length. This can be readily explained by the Einstein law: as the length increases, both translational and rotational drag forces increase, which results in a decrease in both the diffusivity and rotational velocity. Importantly, as the length increases from $1.5 \mu \mathrm{m}$ to $3.5 \mu \mathrm{m}$ the degree of decrease in $D_{r}$ is notably different from that of $D_{r 0}$, while their $D_{t}$ decreases in a similar way that of $D_{t 0}$. In other words, core-shell NWs move much more linearly than predicted (see supporting movie $\mathrm{S} 1$ ). In order to rationalize such a discrepancy in $D_{r}$, we turn our attention to the vortex at the tail of the cathode that was found in the numerical model (see again Figure 2b). These vortices were formed by the convergence of flow from different directions. We postulate that such vortex causes additional fluidic drag that stabilizes the nanowire and contributes to the large reduction in the rotational diffusivity (i.e. higher directionality). To elaborate, for stable propulsion the center of the drag force should be located behind the center of the thrust, and stability increases with the distance between these two centers. In our case, the center of thrust is located at the tip of the anode $(\mathrm{Au})$, while the center of the drag force, which is normally at the center of volume for the nanomotor, is shifted toward the cathode $(\mathrm{Ru})$ due to the additional drag by vortex at the Ru end. The distance between the two centers increases with the size of the vortex as the length increases, which is consistent with our observation that rotational diffusivity decreases with length.

Contrary to intuition and our simulation results, the speeds $(v)$ of core-shell NWs increased with increasing length (Table 1). The instantaneous speeds, analyzed to confirm the MSD result also show the same tendency of speed with length (Figure 3d). Such increase in speed with length was found for micromotors driven by bubble releasing mechahism, ${ }^{55}$ but is completely unexpected for nanorod motors driven by self-electroosmosis. For example, previous 
studies conducted on $\mathrm{Cu} / \mathrm{Pt}$ or $\mathrm{Au} / \mathrm{Pt}$ nanomotors shows a decrease in speed with increasing nanomotor length, most likely due to increasing drag force with increasing length. ${ }^{21,56}$ Such an abnormal increase in speed with length, therefore, hints at an additional driving force that increases with length; it superimposes on the main driving force (electroosmotic force), resulting in an increase in the net propulsive force of the nanomotor (Figure 3e). We propose the extra driving force originated from the self-diffusiophoresis mechanism that is popularly used to explain the motion of Janus particles that produce neutral molecules. ${ }^{26}$ To begin with, besides the electrochemical catalysis of $\mathrm{H}_{2} \mathrm{O}_{2}$ described at the beginning of our discussion, over $99 \%$ of $\mathrm{H}_{2} \mathrm{O}_{2}$ is believed to undergo chemical decomposition on $\mathrm{Ru}$ surface, ${ }^{48}$ producing a large number of oxygen molecules that establishes an oxygen gradient pointing from $\mathrm{Ru}$ to $\mathrm{Au}$. $\mathrm{A}$ diffusiophoretic force, then, acts on the oxygen-producing end in the same way as a $\mathrm{Pt}-\mathrm{SiO}_{2}$ Janus particle moving in $\mathrm{H}_{2} \mathrm{O}_{2}$. We note that a similar geometry of an inert nanowire with a catalytic manganese oxide tip has been reported to show enhanced diffusion in $\mathrm{H}_{2} \mathrm{O}_{2}$, indirectly supporting our hypothesis that oxygen gradient can provide an axial force on a cylindrical geometry. 57

The overall propulsion force for a $\mathrm{Au} / \mathrm{Ru}$ core-shell NW is therefore a vector sum of the diffusiophoretic and electroosmotic force. Since both forces coincidentally point from $\mathrm{Ru}$ to $\mathrm{Au}$ in this case, we then expect the diffusiophoretic component to enhance the overall mobility of the core-shell NW. In addition, since this effect increases with the magnitude of the oxygen gradient, a nanowire with a longer $\mathrm{Ru}$ shell would therefore produces more diffusiophoretic force and leads to faster overall propulsion. This explains the seemingly peculiar trend of motor speed increasing with nanowire length. Although our hypothesis that self-diffusiophoresis due to the generated $\mathrm{O}_{2}$ concentration on the $\mathrm{Ru}$ surface leads to a speed enhancement is intuitive, the exact 
magnitude of such an enhancement remains unknown. It is, therefore, difficult to directly compare the relative strength from the enhanced propulsive force due to diffusiophoresis to the larger viscous drag for a longer rod.

\section{Catalytic propulsion of the core-shell NWs with various material combinations and the} prediction of their propulsive force. We now attempt to generalize the contribution of diffusiophoresis to other core-shell nanowires beyond $\mathrm{Au} / \mathrm{Ru}$. $\mathrm{Rh}$ and $\mathrm{Ru}$ have similar activity towards catalyzing the decomposition of $\mathrm{H}_{2} \mathrm{O}_{2},{ }^{58}$ but $\mathrm{Au} / \mathrm{Ru}$ and $\mathrm{Au} / \mathrm{Rh}$ bimetallic motors move to opposite directions. ${ }^{22}$ Therefore, by replacing $\mathrm{Ru}$ with $\mathrm{Rh}$ we expect to extract more information on how diffusiophoresis influences the motor's behaviors. To this end, we fabricated $\mathrm{Au} / \mathrm{Rh}$ and $\mathrm{Rh} / \mathrm{Au}$ core-shell NWs with a total length of $2.5 \mu \mathrm{m}$. Their motion in the presence of 20 wt $\% \mathrm{H}_{2} \mathrm{O}_{2}$ was compared with that of $\mathrm{Au} / \mathrm{Ru}$ core-shell NWs. The trajectories of the coreshell NWs are represented in Figure 4 (right column).

We found that the speeds of these three types of core-shell nanomotors follow the trend of $\mathrm{Au} / \mathrm{Ru} \gg \mathrm{Rh} / \mathrm{Au}>\mathrm{Au} / \mathrm{Rh}$ (See the Supplementary movie 2), which cannot be explained solely by the existing theory of self-electroosmosis. The speed of a self-electroosmotic nanomotor, in general, is determined by a combination of their surface charges (zeta potential), and the self-generated electric field as a result of the surface electrochemical reactions. To understand the effects of these two factors on the speeds of the three types of motors, we first measured the zeta potential of $\mathrm{Au} / \mathrm{Ru}, \mathrm{Rh} / \mathrm{Au}$ and $\mathrm{Au} / \mathrm{Rh}$ core-shell NWs, all yielding results of approximately $-26 \mathrm{mV}$. The difference of surface charges is therefore eliminated as a possible reason to explain their speed variations. Secondly, given a previous study by Wang et al. ${ }^{22}$ showing similar speeds of $\mathrm{Au} / \mathrm{Ru}, \mathrm{Rh} / \mathrm{Au}$ and $\mathrm{Au} / \mathrm{Rh}$ segmented NW motors, we believe the difference in the self-generated electric field by these three samples is insignificant. In addition, 
one may wonder if the speed differences among these three types of motors could have originated from varying degrees of core protrusion where a small segment of the core metal is exposed, as shown in Figure S4a. However, our simulation results show that the effect of the protrusion is too small to account for the large speed variation. A detailed discussion can be found in the supporting information. To briefly summarize, the self-electroosmotic mechanism predicts similar speeds for these three samples, which is in clear contrast to what we have experimentally observed.

Given the inadequacy of self-electroosmosis in explaining the observed speed trend, we attempt to reconcile our results with self-diffusiophoresis that successfully explained the behaviors of $\mathrm{Au} / \mathrm{Ru}$ core-shell nanomotors. The magnitude of the self-diffusiophoretic force, i.e. the additional driving force, depends on the $\mathrm{O}_{2}$ concentration gradient developed by the decomposition of $\mathrm{H}_{2} \mathrm{O}_{2}$ on the catalytically active layer. According to previous studies, the decomposition rate of $\mathrm{H}_{2} \mathrm{O}_{2}$ on $\mathrm{Ru}$ is twice as high as $\mathrm{Rh}$, while it is ten times lower on $\mathrm{Au}$ than on $\mathrm{Rh}^{58}$ Thus, the predicted magnitude of the self-diffusiophoretic forces on core-shell NWs of equal lengths follows $\mathrm{Au} / \mathrm{Ru} \gg \mathrm{Au} / \mathrm{Rh}>\mathrm{Rh} / \mathrm{Au}$ (shells being $\mathrm{Ru}, \mathrm{Rh}$ and $\mathrm{Au}$, respectively), provided that the total amount of $\mathrm{H}_{2} \mathrm{O}_{2}$ decomposition is defined as the product of the decomposition rate and the reactive surface (shell) area. The diffusiophoretic force directions on $\mathrm{Au} / \mathrm{Rh}, \mathrm{Rh} / \mathrm{Au}$, and $\mathrm{Au} / \mathrm{Ru}$ core-shell nanomotors can be predicted as $\mathrm{Rh}->\mathrm{Au}, \mathrm{Au}->\mathrm{Rh}$ and $\mathrm{Ru}-$ $>\mathrm{Au}$, respectively. The predicted force magnitudes and directions of the above three motors are illustrated in the left column of Figure 4, and compared to experimental results (right column). The relative speeds of these three nanomotors are estimated by comparing the magnitudes of the propulsive forces that is the sum of the electroosmotic and diffusiophoretic forces. Our 
prediction is in reasonable and qualitative agreement with our experimental observation, lending support to the combined propulsion mechanism we proposed.

\section{Conclusions}

In conclusion, $\mathrm{Au} / \mathrm{Ru}$ core-shell NWs have been fabricated by electrodeposition and studied as a catalytic nanomotor when submerged in $\mathrm{H}_{2} \mathrm{O}_{2}$. Their maximum speed was observed to be $41 \pm 7.6 \mu \mathrm{m} / \mathrm{s}$ in $20 \mathrm{wt} \% \mathrm{H}_{2} \mathrm{O}_{2}$. Decreased rotational diffusivity or, equivalently, enhanced directionality is observed with increased length, which likely results from the formation of vortices at the Ru end of the nanowire, as revealed by numerical simulation. Interestingly, this nanomotor design exhibits a positive length-speed correlation, which is contrary to the previously reported rod-shaped nanomotor systems or our own simulation results. We reconcile such a conflict by hypothesizing that the overall propulsion originates from two simultaneous mechanisms, namely self-diffusiophoresis and self-electroosmosis. For a core-shell Au/Ru NW, the self-diffusiophoretic force, originated from the oxygen produced at $\mathrm{Ru}$ surface, is superimposed on the self-electroosmotic force in the same direction, increasing the net motor speed. In addition, when the catalytic active surface area $(\mathrm{Ru})$ increases with length, the selfdiffusiophoretic force increases due to a higher $\mathrm{O}_{2}$ concentration gradient, leading to a higher motor speed. The proposed propulsion mechanism also justifies the large difference in speed among $\mathrm{Au} / \mathrm{Rh}, \mathrm{Rh} / \mathrm{Au}$ and $\mathrm{Au} / \mathrm{Ru}$ core-shell NWs, which is not observed for conventional bimetallic NWs of similar compositions. Through careful experimental designs and the adopted fabrication strategy, we reveal the possible role of self-diffusiophoresis on propelling a widely studied nanomotor system. Although the exact magnitude of this force remains yet to be uncovered, we have reason to believe that this finding will advance our understanding of forces 
on nanomotors and help reconcile non-cooperating experimental results. Self-diffusiophoresis, although popular in other nanomotors systems, has been traditionally ignored in bimetallic nanomotors. We are once again reminded of the complex interplay between diffusiophoresis and electroosmosis, and call for a renewed attention on this matter. ${ }^{59}$

\section{Methods/Experimental}

AAO-Si wafer bonding: A platform for the fabrication of core-shell nanowires. An AAO (Anodisc 25, $200 \mathrm{~nm}$, Whatman, $200 \mathrm{~nm}$ ) membrane and Si chip (2 cm x2 cm) were sonicated in acetone, isopropanol and distilled (DI) water for $10 \mathrm{~min}$ to remove organic residuals. An additional cleaning process was conducted for the $\mathrm{Si}$ chip. This consists of dipping and sonicating the Si chip in piranha solution $\left(7: 3 \mathrm{H}_{2} \mathrm{SO}_{4}: \mathrm{H}_{2} \mathrm{O}_{2}\right)$ for $10 \mathrm{~min}$; Note that the piranha solution is a strong oxidizer, which requires great care in handling. The cleaned AAO and Si chip were further coated with a Au layer of a thickness of $500 \mathrm{~nm}$ by means of thermal evaporation (EDWARD, UK) at $1 \times 10^{-6}$ mTorr. The coated AAO was, then, transferred to the coated Si substrate via $\mathrm{Au} / \mathrm{Au}$ compressive bonding at $1 \times 10^{-5} \mathrm{mTorr}, 1 \mathrm{MPascal}$ and $320^{\circ} \mathrm{C}$ for $15 \mathrm{~min}$.

Fabrication of $\mathbf{A u} / \mathbf{R u}$ core-shell. The bonded AAO membrane was then subjected to electrodeposition to grow $\mathrm{Au} / \mathrm{Ru}$ core-shell nanowires as follows. $\mathrm{Au}$ nanowires were electrochemically deposited in the nanopores of AAO at $-2 \mathrm{~mA} \mathrm{~cm}{ }^{-2}$ for $90 \mathrm{~min}$. The Au bath consisted of gold (I) potassium cyanide $\left(\mathrm{AuK}(\mathrm{CN})_{2}, 8 \mathrm{~g} \mathrm{~L}^{-1}\right)$, citric acid $\left(\mathrm{C}_{6} \mathrm{H}_{8} \mathrm{O}_{7}, 90 \mathrm{~g} \mathrm{~L}^{-1}\right)$, potassium citrate $\left(\mathrm{C}_{6} \mathrm{H}_{5} \mathrm{~K}_{3} \mathrm{O}_{7}, 90 \mathrm{~g} \mathrm{~L}^{-1}\right)$ and $10 \mathrm{~mL} \mathrm{~L}^{-1}$ of a brightener concentrate which contained cobalt (II) carbonate as a grain refiner $\left(0.05 \mathrm{~g} \mathrm{~L}^{-1}\right)$. After the core deposition, the AAO membrane was selectively etched in $5 \mathrm{M} \mathrm{NaOH}$ for 2 hours, resulting in the formation of 
freestanding Au nanowire arrays on the Si substrate. After etching the AAO, the standing array was thoroughly rinsed with DI water to remove residuals for Ru shell deposition. The shell was galvanostatically deposited at $-0.5 \mathrm{mAcm}^{-2}$ for $10 \mathrm{~min}$ in a $\mathrm{Ru}$ bath (pH 1.2). The bath consisted of $0.05 \mathrm{M}$ of $\mathrm{RuCl}_{3}$ and the $\mathrm{pH}$ of the bath was adjusted by adding $\mathrm{HCl}$ (37 wt.\%). After the $\mathrm{Ru}$ deposition, the $\mathrm{Au} / \mathrm{Ru}$ core-shell nanowires were released by sonication in DI water.

Characterization. Scanning electron microscopy (SEM) images were acquired by a backscattering electron detector in Zeiss ULTRA 55. High-angle annular dark field (HAADF) scanning transmission electron microscopy (STEM) images and energy dispersive X-ray (EDX) maps were acquired using FEI Talos F200X under an accelerating voltage of 200kV.

Manipulation. We investigated catalytic locomotion of our core-shell nanowires in $\mathrm{H}_{2} \mathrm{O}_{2}$ under an inverted optical microscope equipped with an objective lens of $40 \mathrm{X}$. Image sequences were recorded using a CCD camera at $30 \mathrm{FPS}$.

Numerical modelling. The numerical modelling was performed using the commercial multiphysics simulation package COMSOL (5.1). In the simulation, 2D axisymmetric model was selected, and geometry of a nanomotor was defined as a simple rod that has a diameter of 300 $\mathrm{nm}$ and a length of $2.5 \mu \mathrm{m}$. To study the length effect, the length of rod varied as $0.5,1.5,2.5$ and $3.5 \mu \mathrm{m}$. Diluted species transport, electrostatic, and creeping flow models were coupled to compute proton concentration, electric potential, and fluid flow around the core-shell nanowire. The size of mesh was selected to be $1 \mathrm{e}-6 \mu \mathrm{m}$ and $5 \mathrm{e}-7 \mu \mathrm{m}$ for maximum and minimum element 
size, respectively. The detailed physics and boundary conditions are noted in supporting information S2.

Table 1. Measured parameters $\left(D_{t}, v, D_{r}\right.$ and $\left.\omega\right)$ and theoretical values $\left(D_{t 0}\right.$ and $\left.D_{r 0}\right)$ of Au/Ru core-shell NWs. The errors are obtained from the fitting.

\begin{tabular}{|c|c|c|c|c|c|c|}
\hline Length & $D_{t}\left(\mu \mathrm{m}^{2} / \mathrm{s}\right)$ & $\begin{array}{c}D_{t 0} \\
\left(\mu \mathrm{m}^{2} / \mathrm{s}\right)\end{array}$ & $v(\mu \mathrm{m} / \mathrm{s})$ & $D_{r}\left(\mu \mathrm{m}^{2} / \mathrm{rad}\right)$ & $\begin{array}{c}D_{r 0} \\
\left(\mu \mathrm{m}^{2} / \mathrm{rad}\right)\end{array}$ & $\omega(\mathrm{rad} / \mathrm{s})$ \\
\hline $1.5 \mu \mathrm{m}$ & $1.18 \pm 0.11$ & 0.79 & $11.23 \pm 1.30$ & $2.36 \pm 0.10$ & 1.50 & $4.91 \pm 0.03$ \\
\hline $2.5 \mu \mathrm{m}$ & $1.16 \pm 0.23$ & 0.61 & $19.80 \pm 0.31$ & $0.65 \pm 0.02$ & 0.45 & $3.43 \pm 0.01$ \\
\hline $3.5 \mu \mathrm{m}$ & $1.05 \pm 0.28$ & 0.50 & $30.12 \pm 0.25$ & $0.17 \pm 0.01$ & 0.20 & $1.12 \pm 0.01$ \\
\hline
\end{tabular}




\section{References}

(1) Ismagilov, R. F.; Schwartz, A.; Bowden, N.; Whitesides, G. M. Autonomous Movement and Self-Assembly. Angew. Chem. Int. Ed. Engl. 2002, 41, 652-654.

(2) Wang, W.; Duan, W.; Ahmed, S.; Mallouk, T. E.; Sen, A. Small Power: Autonomous Nanoand Micromotors Propelled by Self-Generated Gradients. Nano Today 2013, 8, 531-554.

(3) Mano, N.; Heller, A. Bioelectrochemical Propulsion. J. Am. Chem. Soc. 2005, 127, 1157411575.

(4) Ma, X.; Jannasch, A.; Albrecht, U. R.; Hahn, K.; Miguel-Lopez, A.; Schaffer, E.; Sanchez,

S. Enzyme-Powered Hollow Mesoporous Janus Nanomotors. Nano Lett. 2015, 15, 7043-7050.

(5) Gaspar, S. Enzymatically Induced Motion at Nano- and Micro-Scales. Nanoscale 2014, 6, 7757-7763.

(6) Wang, H.; Pumera, M. Fabrication of Micro/Nanoscale Motors. Chem. Rev. 2015, 115, 8704-8735.

(7) Li, J.; Shklyaev, O. E.; Li, T.; Liu, W.; Shum, H.; Rozen, I.; Balazs, A. C.; Wang, J. SelfPropelled Nanomotors Autonomously Seek and Repair Cracks. Nano Lett. 2015, 15, 7077-7085.

(8) Dey, K. K.; Zhao, X.; Tansi, B. M.; Mendez-Ortiz, W. J.; Cordova-Figueroa, U. M.;

Golestanian, R.; Sen, A. Micromotors Powered by Enzyme Catalysis. Nano Lett. 2015, 15, 83118315.

(9) Li, J.; Gao, W.; Dong, R.; Pei, A.; Sattayasamitsathit, S.; Wang, J. Nanomotor Lithography. Nat. Commun. 2014, 5, 5026.

(10) Kline, T. R.; Paxton, W. F.; Wang, Y.; Velegol, D.; Mallouk, T. E.; Sen, A. Catalytic Micropumps: Microscopic Convective Fluid Flow and Pattern Formation. J. Am. Chem. Soc. 2005, 127, 17150-17151. 
(11) Sundararajan, S.; Lammert, P. E.; Zudans, A. W.; Crespi, V. H.; Sen, A. Catalytic Motors for Transport of Colloidal Cargo. Nano Lett. 2008, 8, 1271-1276.

(12) Dey, K. K.; Wong, F.; Altemose, A.; Sen, A. Catalytic Motors-Quo Vadimus? Curr. Opin. Colloid Interface Sci. 2016, 21, 4-13.

(13) Yadav, V.; Duan, W.; Butler, P. J.; Sen, A. Anatomy of Nanoscale Propulsion. Annu. Rev. Biophys. 2015, 44, 77-100.

(14) Sanchez, S.; Soler, L.; Katuri, J. Chemically Powered Micro- and Nanomotors. Angew. Chem. Int. Ed. Engl. 2015, 54, 1414-1444.

(15) Wang, W.; Duan, W.; Ahmed, S.; Sen, A.; Mallouk, T. E. From One to Many: Dynamic Assembly and Collective Behavior of Self-Propelled Colloidal Motors. Acc. Chem. Res. 2015, 48, 1938-1946.

(16) Guix, M.; Mayorga-Martinez, C. C.; Merkoci, A. Nano/Micromotors in (Bio)Chemical Science Applications. Chem. Rev. 2014, 114, 6285-6322.

(17) Zhou, C.; Zhang, H.; Li, Z.; Wang, W. Chemistry Pumps: A Review of Chemically Powered Micropumps. Lab Chip 2016, 16, 1791-1811.

(18) Colberg, P. H.; Reigh, S. Y.; Robertson, B.; Kapral, R. Chemistry in Motion: Tiny Synthetic Motors. Acc. Chem. Res. 2014, 47, 3504-3511.

(19) Solovev, A. A.; Mei, Y.; Bermudez Urena, E.; Huang, G.; Schmidt, O. G. Catalytic Microtubular Jet Engines Self-Propelled by Accumulated Gas Bubbles. Small 2009, 5, 16881692.

(20) Huang, W.; Manjare, M.; Zhao, Y. Catalytic Nanoshell Micromotors. J. Phys. Chem. C 2013, 117, 21590-21596. 
(21) Liu, R.; Sen, A. Autonomous Nanomotor Based on Copper-Platinum Segmented Nanobattery. J. Am. Chem. Soc. 2011, 133, 20064-20067.

(22) Wang, Y.; Hernandez, R. M.; Bartlett, D. J.; Bingham, J. M.; Kline, T. R.; Sen, A.;

Mallouk, T. E. Bipolar Electrochemical Mechanism for the Propulsion of Catalytic Nanomotors in Hydrogen Peroxide Solutions†. Langmuir 2006, 22, 10451-10456.

(23) Pumera, M. Electrochemically Powered Self-Propelled Electrophoretic Nanosubmarines. Nanoscale 2010, 2, 1643-1649.

(24) Ma, X.; Hahn, K.; Sanchez, S. Catalytic Mesoporous Janus Nanomotors for Active Cargo Delivery. J. Am. Chem. Soc. 2015, 137, 4976-4979.

(25) Yamamoto, D.; Takada, T.; Tachibana, M.; Iijima, Y.; Shioi, A.; Yoshikawa, K. Micromotors Working in Water through Artificial Aerobic Metabolism. Nanoscale 2015, 7, 13186-13190.

(26) Howse, J. R.; Jones, R. A.; Ryan, A. J.; Gough, T.; Vafabakhsh, R.; Golestanian, R. SelfMotile Colloidal Particles: From Directed Propulsion to Random Walk. Phys. Rev. Lett. 2007, $99,048102$.

(27) Frankel, A. E.; Khair, A. S. Dynamics of a Self-Diffusiophoretic Particle in Shear Flow. Phys. Rev. E: Stat., Nonlinear, Soft Matter Phys. 2014, 90, 013030.

(28) Golestanian, R.; Liverpool, T. B.; Ajdari, A. Propulsion of a Molecular Machine by Asymmetric Distribution of Reaction Products. Phys. Rev. Lett. 2005, 94, 220801.

(29) Ma, X.; Jang, S.; Popescu, M. N.; Uspal, W. E.; Miguel-López, A.; Hahn, K.; Kim, D.-P.; Sánchez, S. Reversed Janus Micro/Nanomotors with Internal Chemical Engine. ACS Nano 2016, $10,8751-8759$. 
(30) Wilson, D. A.; Nolte, R. J.; van Hest, J. C. Autonomous Movement of Platinum-Loaded Stomatocytes. Nat. Chem. 2012, 4, 268-274.

(31) Wang, S.; Wu, N. Selecting the Swimming Mechanisms of Colloidal Particles: Bubble Propulsion Versus Self-Diffusiophoresis. Langmuir 2014, 30, 3477-3486.

(32) Hu, J.; Odom, T. W.; Lieber, C. M. Chemistry and Physics in One Dimension: Synthesis and Properties of Nanowires and Nanotubes. Acc. Chem. Res. 1999, 32, 435-445.

(33) Nicewarner-Pena, S. R.; Freeman, R. G.; Reiss, B. D.; He, L.; Pena, D. J.; Walton, I. D.;

Cromer, R.; Keating, C. D.; Natan, M. J. Submicrometer Metallic Barcodes. Science 2001, 294, $137-141$.

(34) Jang, B.; Pellicer, E.; Guerrero, M.; Chen, X.; Choi, H.; Nelson, B. J.; Sort, J.; Pane, S. Fabrication of Segmented $\mathrm{Au} / \mathrm{Co} / \mathrm{Au}$ Nanowires: Insights in the Quality of $\mathrm{Co} / \mathrm{Au}$ Junctions. ACS Appl. Mater. Interfaces 2014, 6, 14583-14589.

(35) Ozel, T.; Bourret, G. R.; Mirkin, C. A. Coaxial Lithography. Nat. Nanotechnol. 2015, 10, 319-324.

(36) Kline, T. R.; Paxton, W. F.; Mallouk, T. E.; Sen, A. Catalytic Nanomotors: RemoteControlled Autonomous Movement of Striped Metallic Nanorods. Angew. Chem. Int. Ed. Engl. 2005, $117,754-756$.

(37) Hangarter, C. M.; Lee, Y. I.; Hernandez, S. C.; Choa, Y. H.; Myung, N. V. Nanopeapods by Galvanic Displacement Reaction. Angew. Chem. Int. Ed. Engl. 2010, 49, 7081-7085.

(38) Lauhon, L. J.; Gudiksen, M. S.; Wang, D.; Lieber, C. M. Epitaxial Core-Shell and CoreMultishell Nanowire Heterostructures. Nature 2002, 420, 57-61. 
(39) Hwang, J.; Min, B.; Lee, J. S.; Keem, K.; Cho, K.; Sung, M. Y.; Lee, M. S.; Kim, S. Al2o3 Nanotubes Fabricated by Wet Etching of Zno/Al2o3 Core/Shell Nanofibers. Adv. Mater. 2004, $16,422-425$.

(40) Yin, Y.; Lu, Y.; Sun, Y.; Xia, Y. Silver Nanowires Can Be Directly Coated with Amorphous Silica to Generate Well-Controlled Coaxial Nanocables of Silver/Silica. Nano Lett. 2002, 2, 427-430.

(41) Kong, X. Y.; Ding, Y.; Wang, Z. L. Metal-Semiconductor Zn-Zno Core-Shell Nanobelts and Nanotubes. J. Phys. Chem. B 2004, 108, 570-574.

(42) Cao, H. Q.; Xu, Z.; Sang, H.; Sheng, D.; Tie, C. Y. Template Synthesis and Magnetic Behavior of an Array of Cobalt Nanowires Encapsulated in Polyaniline Nanotubules. Adv. Mater. 2001, 13, 121-123.

(43) Hu, J. Q.; Meng, X. M.; Jiang, Y.; Lee, C. S.; Lee, S. T. Fabrication of Germanium-Filled Silica Nanotubes and Aligned Silica Nanofibers. Adv. Mater. 2003, 15, 70-73.

(44) Jang, B.; Chen, X.-Z.; Siegfried, R.; Montero Moreno, J. M.; Özkale, B.; Nielsch, K.;

Nelson, B. J.; Pané, S. Silicon-Supported Aluminum Oxide Membranes with Ultrahigh Aspect Ratio Nanopores. RSC Adv. 2015, 5, 94283-94289.

(45) Hill, J. J.; Haller, K.; Gelfand, B.; Ziegler, K. J. Eliminating Capillary Coalescence of Nanowire Arrays with Applied Electric Fields. ACS Appl. Mater. Interfaces 2010, 2, 1992-1998. (46) Moran, J. L.; Wheat, P. M.; Posner, J. D. Locomotion of Electrocatalytic Nanomotors Due to Reaction Induced Charge Autoelectrophoresis. Phys. Rev. Lett. E 2010, 81, 065302.

(47) Moran, J. L.; Posner, J. D. Electrokinetic Locomotion Due to Reaction-Induced Charge Auto-Electrophoresis. J. Fluid Mech. 2011, 680, 31-66. 
(48) Wang, W.; Chiang, T. Y.; Velegol, D.; Mallouk, T. E. Understanding the Efficiency of Autonomous Nano- and Microscale Motors. J .Am. Chem. Soc. 2013, 135, 10557-10565. (49) Marine, N. A.; Wheat, P. M.; Ault, J.; Posner, J. D. Diffusive Behaviors of CircleSwimming Motors. Phys. Rev. Lett. E 2013, 87.

(50) Lee, T. C.; Alarcon-Correa, M.; Miksch, C.; Hahn, K.; Gibbs, J. G.; Fischer, P. SelfPropelling Nanomotors in the Presence of Strong Brownian Forces. Nano Lett. 2014, 14, 2407 2412.

(51) Demirok, U. K.; Laocharoensuk, R.; Manesh, K. M.; Wang, J. Ultrafast Catalytic Alloy Nanomotors. Angew. Chem. Int. Ed. Engl. 2008, 47, 9349-9351.

(52) Laocharoensuk, R.; Burdick, J.; Wang, J. Carbon-Nanotube-Induced Acceleration of Catalytic Nanomotors. ACS Nano 2008, 2, 1069-1075.

(53) Ebbens, S.; Jones, R. A.; Ryan, A. J.; Golestanian, R.; Howse, J. R. Self-Assembled Autonomous Runners and Tumblers. Phys. Rev. E: Stat., Nonlinear, Soft Matter Phys. 2010, 82, 015304.

(54) Charsooghi, M. A.; Akhlaghi, E. A.; Tavaddod, S.; Khalesifard, H. R. A Matlab Program to Calculate Translational and Rotational Diffusion Coefficients of a Single Particle. Comput. Phys. Commun. 2011, 182, 400-408.

(55) Wang, H.; Moo, J. G.; Pumera, M. From Nanomotors to Micromotors: The Influence of the Size of an Autonomous Bubble-Propelled Device Upon Its Motion. ACS Nano 2016, 10, 50415050.

(56) Dhar, P.; Fischer, T. M.; Wang, Y.; Mallouk, T. E.; Paxton, W. F.; Sen, A. Autonomously Moving Nanorods at a Viscous Interface. Nano Lett. 2006, 6, 66-72. 
(57) Morgan, A. R.; Dawson, A. B.; McKenzie, H. S.; Skelhon, T. S.; Beanland, R.; Franks, H. P. W.; Bon, S. A. F. Chemotaxis of Catalytic Silica-Manganese Oxide "Matchstick" Particles. Mater. Horiz. 2014, 1, 65-68.

(58) McKee, D. W. Catalytic Decomposition of Hydrogen Peroxide by Metals and Alloys of the Platinum Group. J. Catal. 1969, 14, 355-364.

(59) Brown, A.; Poon, W. Ionic Effects in Self-Propelled Pt-Coated Janus Swimmers. Soft

Matter 2014, 10, 4016-4027. 
ASSOCIATED CONTENT

Supporting Information.

Numerical modelling procedures and additional numerical modelling results of the effect of core protrusion on the speed of nanomotors and supporting figures (PDF)

Supporting movies S1 and S2 (AVI)

This material is available free of charge via the Internet at http://pubs.acs.org.

\section{AUTHOR INFORMATION}

Corresponding Author

*E-mail: vidalp@ethz.ch

*E-mail: bnelson@ethz.ch

\section{Author Contributions}

S.P. and B.J.N. initiated the project. S.P. and B.J. designed the fabrication experiments. B.J. and S.W. fabricated the magnetic nanoswimmers and analyzed their motion. W.W. and B.J. conducted the simulation experiments. B.J., W.W., A.P., X.C., A.H., B.J.N., D.F. and A.F. performed the analysis of the swimmers and provided theoretical discussion. B.J., W.W., A.P., X.C., S.P., and B.J.N. supervised the work and gave critical input. All authors contributed to discussions.

Notes

The authors declare no competing financial interests. 


\section{ACKNOWLEDGMENTS}

Funding support from the European Community’s Seventh Framework Programme (FP7/20072013) under grant agreement 296679 (MANAQA) is acknowledged. S.P. acknowledges financial support by the European Research Council Starting Grant "Magnetoelectric Chemonanomotorics for Chemical and Biomedical Applications (ELECTROCHEMBOTS)", by the ERC grant agreement n. 336456. W.W. is grateful for the financial support from National Natural Science Foundation of China (Grant No. 11402069) and the city government of Shenzhen (Grant No. KQCX20140521144102503). We especially thank Prof. Mahmut Selman Sakar from the Mechanical Engineering (School of Engineering, EPFL), and Carlos C.J. Alcantara from the Multi-Scale Robotics Lab (ETH Zürich) for constructive discussions. 